\title{
ON THE THEORY OF KERNELS OF SCHWARTZ ${ }^{1}$
}

\section{LEON EHRENPREIS}

Let $D$ denote the space of indefinitely differentiable functions of compact carrier on Euclidean space $R$ of dimension $p$, and denote by $D^{\prime}$ the dual of $D$, that is, $D^{\prime}$ is the space of distributions on $R$; we give $D$ and $D^{\prime}$ their usual topologies (see [2]). By ${ }_{2} D,{ }_{2} D^{\prime}$ we denote respectively the spaces corresponding to $D$ and $D^{\prime}$ on $R \times R$. Let $L$ be any continuous linear map of $D \rightarrow D^{\prime}$; then L. Schwartz has shown (see [3] for a summary of Schwartz' results, the details will appear in a series of articles in the Journal D'Analyse (Jerusalem)) that $L$ can be represented by a kernel, i.e. there exists a distribution $S$ on $R \times R$ so that we may write (symbolically)

$$
(L f)(x)=\int S(x, y) f(y) d y
$$

for any $f \in D$. The purpose of this paper is to give a simple proof of this fact and to prove the following result: Let us give the space of continuous linear mappings of $D \rightarrow D^{\prime}$ the compact-open topology (see [1]); then this topology is the same as that of the space ${ }_{2} D^{\prime}$. We shall also obtain an explicit expression for the topology of the space $D_{X}$ of continuous linear maps of $D^{\prime}$ into $D$ with the compactopen topology. The elements of $D_{x}$ are those of ${ }_{2} D$, but the topology of ${ }_{2} D$ is stronger than that of $D_{X}$. It is possible to extend the above methods and results to other spaces, e.g. the space $\mathcal{E}$ of Schwartz. Moreover, the methods and results can be easily extended to indefinitely differentiable manifolds and double coset spaces of Lie groups (cf. Remark 1 following the proof of Theorem 1).

LEMma 1. Let $B$ be a bounded set in ${ }_{2} D$. Then we can find a bounded set $B^{\prime} \subset D$ and $a b>0$ so that every $f \in B$ can be written in the form $\sum \lambda_{i} g_{i} \times h_{i}$ where $\sum\left|\lambda_{i}\right| \leqq b$, and $g_{i}, h_{i} \in B$, and where the series converge in $2 D$.

(For any $g, h \in D, g \times h \in_{2} D$ is the function $(g \times h)(x, y)=g(x) h(y)$.)

Proof. We know (see [2]) that all functions of $B$ have their carriers in a fixed compact set $K$ of $R \times R$ which we may assume to be the cube center origin, side $a-1$. For each $f \in B$, denote by $f^{*}$ the function on $R \times R$ which is equal to $f$ in $K$ and is periodic with period $a$ on

Received by the editors May 24, 1955 and, in revised form, August 17, 1955.

1 Work supported by National Science Foundation Grant NSF5-G1010. 
$R \times R$, and write $B^{*}=\left\{f^{*}\right\}_{f \in B}$, so $B^{*}$ is bounded in the space ${ }_{2} D^{*}$ of indefinitely differentiable periodic functions on $R \times R$. Thus (see[2, Chapter VII, §1]) we may write, for each $f^{*} \in B^{*}$,

$$
f^{*}(x)=\sum c_{j} \exp ((2 \pi i / a) j \cdot x)
$$

where the sum runs over all tuples of the form $j=\left(r_{1} a, r_{2} a, \cdots, r_{2 p} a\right)$, $r_{1}, \cdots, r_{2 p}$ being integers. Moreover, in (1) the numbers $c_{j}$ are rapidly decreasing, uniformly for $f^{*} \in B^{*}$, i.e. for any polynomial $Q$ in $2 p$ variables,

$$
\sup _{j \in B, j}\left|Q(j) c_{j}\right|<\infty .
$$

It is obvious that the numbers $\left(\left|c_{j}\right|^{1 / 3}\right)$ are also uniformly rapidly decreasing for $f \in B$. Let $t \in D$ satisfy

$$
t(x)=\left\{\begin{array}{l}
1 \text { for } x \in \text { cube center origin, side } a-1, \\
0 \text { for } x \text { outside cube center origin, side } a .
\end{array}\right.
$$

Write $j=\left(j_{1}, j_{2}\right)$ where $j_{1}=\left(r_{1} a, \cdots, r_{p} a\right), j_{2}=\left(r_{p+1} a, \cdots, r_{2 p} a\right)$. Then it is obvious that the decomposition

(2) $f=\sum c_{j}\left|c_{j}\right|^{-2 / 3} t \exp \left(2 \pi i / a j_{1} \cdot\right)\left|c_{j}\right|^{1 / 3} \times\left. t \exp \left(2 \pi i / a j_{2} \cdot\right)\left|c_{j} \cdot\right|\right|^{1 / 3}$

has the desired properties of Lemma 1. (It is understood that if $c_{j}=0$, no term appears in the sum in (2).)

Now, let $J$ denote the space of continuous linear maps of $D \rightarrow D^{\prime}$ with the compact-open topology. For any $L \in J, T \in \mathcal{E}^{\prime}$, define $L \# T$ by $(L \# T)(h)=L(h) * T$, where * denotes convolution (see [2, Chapter VI]), for any $h \in D$.

Lemмa 2. $L, T \rightarrow L \# T$ is a separately continuous bilinear map of $J \times \mathcal{E}^{\prime} \rightarrow J$.

(A map $s: V \times W \rightarrow Z$, where $V, W, Z$ are locally convex topological vector spaces, is called separately continuous if $v \rightarrow s\left(v, w^{\prime}\right)$ and $w \rightarrow s\left(v^{\prime}, w\right)$ are equicontinuous as long as $v^{\prime}$ and $w^{\prime}$ remain in bounded sets of $V$ and $W$ respectively.)

Proof. It is clear that $L \# T \in J$. Moreover, if $L_{\alpha} \rightarrow 0$ in $J$, then $L_{\alpha}(g) \rightarrow 0$ in $D^{\prime}$ uniformly for $g$ in any bounded set $B$ of $D$. This implies that $L_{\alpha}(g) * T \rightarrow 0$ in $D^{\prime}$ uniformly for $T$ in any bounded set $B^{\prime}$ of $\mathcal{E}^{\prime}$ and $g \in B$, which means that $L_{\alpha} \# T \rightarrow 0$ in $J$ uniformly for $T \in B^{\prime}$. This proves the first half of Lemma 2 .

On the other hand, suppose $T_{\alpha} \rightarrow 0$ in $\mathcal{E}^{\prime}$; let $B$ be a bounded set in $D$ and $B^{\prime}$ a bounded set in $J$. Then it is clear from the definition of the topology of $J$ that $\{L(h)\}_{L \in B^{\prime}, h \in B}$ is bounded in $D^{\prime}$. Thus, by the continuity of convolution (see $[2$, Chapter VI $]$ ), 


$$
\left(L \# T_{\alpha}\right)(h)=L(h) * T_{\alpha} \rightarrow 0 \text { in } D^{\prime}
$$

uniformly for $L \in B^{\prime}, h \in B$. By the definition of the topology of $J$, this means $L \# T_{\alpha} \rightarrow 0$ in $J$ uniformly for $L \in B^{\prime}$. This concludes the proof of Lemma 2.

Let us consider first the space $\mathcal{K}$ of continuous linear maps of $D \rightarrow \mathcal{E}$, with the compact-open topology. That any $U \in \mathcal{K}$ is defined by a kernel is not difficult to show: For any $x \in R, f \in D$, set $U_{x}(f)$ $=(U(f))(x)$. Now, for each $f, U(f) \in \mathcal{E}$, so $x \rightarrow U_{x}(f)$ is an indefinitely differentiable function. We define the kernel $U^{*}$ by $U^{*}(h)$ $=\int U_{x}\left(h_{X_{2}=x}\right) d x$ for any $h \in_{2} D$ where the integration is over $R$ and where $h_{X_{2}=x}$ is the function on $R: y \rightarrow h(y, x)$. It is easily verified that $U^{*}$ defines a continuous linear function on ${ }_{2} D$, i.e. $U^{*} \in \in_{2} D^{\prime}$.

For any $S \in_{2} D^{\prime}, L \in J$, we say that $S$ is a kernel representing $L$ if, for any $g, h \in D, S(g \times h)=(L(g))(h)$. It is easily verified that $U^{*}$ is a kernel representing $U$. Moreover, we can easily establish by the method of proof of Lemma 2,

LEMMA 3. $L, f \rightarrow L \# f$ is a separately continuous bilinear map of $J \times D \rightarrow \mathfrak{K}$.

We can now prove our main result:

THEOREM 1. Let $t:{ }_{2} D^{\prime} \rightarrow J$ be the map which assigns to each $S \in_{2} D^{\prime}$ the $L \in J$ such that $S$ is a kernel representing $L$. Then $t$ is a topological isomorphism onto.

Proof. It is clear that $t$ is a continuous linear map. Moreover, since $D \times D$ is dense in ${ }_{2} D$ (see [2]), $t$ is one-one. Now, if $\delta \in \mathcal{E}^{\prime}$ is the Dirac measure, i.e. the measure representing the mass of +1 placed at the origin, then it is clear that, for any $L \in J, L \# \delta=L$. Now, let $\left\{f_{j}\right\}$ be a sequence in $D$ such that $f_{j} \rightarrow \delta$ in $\mathcal{E}^{\prime}$. By Lemma 2 , it follows that $L \# f_{j} \rightarrow L$ in $J$. But, by Lemma 3 , each $L \# f_{j}$ lies in $\mathcal{K}$, so that $L \# f_{j}$ lies in the image $t\left({ }_{2} D^{\prime}\right)$ by our remarks preceding Lemma 3. We conclude that $t\left({ }_{2} D^{\prime}\right)$ is dense in $J$. On the other hand, ${ }_{2} D^{\prime}$ is known to be complete. Thus, to establish Theorem 1 , it remains to show that $t^{-1}$ is a continuous linear map of $t\left({ }_{2} D^{\prime}\right)$ (with topology induced from $J$ ) into ${ }_{2} D^{\prime}$.

Since $t^{-1}$ is linear, we need prove continuity at zero only. Suppose that $S_{\alpha} \in_{2} D^{\prime}, t\left(S_{\alpha}\right) \rightarrow 0$ in $J$. Then it follows that $S_{\alpha}(g \times h) \rightarrow 0$ uniformly for $g, h$ in any bounded sets of $D$. It results immediately from Lemma 1 that $S_{\alpha}(f) \rightarrow 0$ uniformly for $f$ in any bounded set of ${ }_{2} D$, i.e. $S_{\alpha} \rightarrow 0$ in ${ }_{2} D^{\prime}$. This proves the continuity of $t^{-1}$ and completes the proof of Theorem 1.

REMARK 1. Lemma 1 is the only part of the proof of Theorem 1 that 
uses special properties of the space $D$, and, in fact, the analog of Theorem 1 holds for (essentially) all function spaces for which an analog of Lemma 1 can be found. For the space $\mathcal{E}$, the result corresponding to Lemma 1 can be proved by use of Lemma 1 and a suitable "partition of unity." For the space $S$ of Schwartz (see [2]) the corresponding result can be proven by use of series in Hermite functions instead of the Fourier series that were used in the proof of Lemma 1. For differential forms on an indefinitely differential manifold, the result corresponding to Lemma 1 can be proved by use of a suitable "partition of unity." Of course, the operation \# is not defined for currents, but it can be replaced by a local form of convolution. Finally, the result for double coset spaces of Lie groups can be proved by use of suitable projections (see [1]). All the above results are special cases of a general theorem of A. Grothendieck, announced in Ann. Inst. Fourier vol. 4 (1952) pp. 73-112, especially pp. 78 and 105.

REMARK 2. Instead of considering maps of $D \rightarrow D^{\prime}$, we could consider maps of $D \rightarrow D_{1}^{\prime}$, where $D_{1}^{\prime}$ is a space of distributions on another Euclidean space. The result and its proof are exactly as in the previous case.

We shall show that for maps of $D^{\prime} \rightarrow D$, the result is somewhat different from what we might expect from the above. Let, then, $V$ be the space of continuous linear maps of $D^{\prime} \rightarrow D$ with the compactopen topology. For any $x \in R, U \in V$, let $U_{x}$ be the function on $\mathfrak{D}^{\prime}: U_{x}(T)=[U(T)](x)$. It is obvious that $U_{x}$ is continuous on $D^{\prime}$ so, by the reflexivity of $D, U_{x} \in D$. Write $U_{x}(y)=r(x, y)$ for any $y \in R$. For any $a \in R$ we denote by $\delta_{a}$ the measure representing a mass of +1 at the point $a$. Then it is clear that, for any $x \in R$,

$$
\left\{U_{x}\left(\delta_{a}\right)\right\}_{a} \in R=\{r(x, a)\}_{a \in R}
$$

is bounded in $D$, and, moreover, these sets are uniformly bounded in $D$ for $x \in R$. It follows that $r \in \in_{2} D$. We call $r$ the kernel representing $U$.

Conversely, any $r \in_{2} D$ defines the continuous linear map $U: T \rightarrow f$ where $f(x)=T\left(r_{X_{1}=x}\right)$, and it is clear that $r$ is just the kernel representing $U$.

We define a new topology on the set of functions of ${ }_{2} D$ : For each non-negative integer $j$ we choose two finite sequences of constantcoefficient differential operators $Q_{j 1}^{(1)}, Q_{j 2}^{(1)}, \ldots, Q_{j i_{j}}^{(1)}$, and $Q_{j 1}^{(2)}$, $Q_{j 2}^{(2)}, \cdots, Q_{j k_{j}}^{(2)}$ on $R$; then call $N$ the set of $f \in_{2} D$ which satisfy, for each $j, l$,

$$
\max _{|x| \geq j,|y| \geq^{l}}\left|\left(Q_{j m}^{(1)} Q_{l t}^{(2)} f\right)\right|(x, y) \leqq 1
$$


for $m=1,2, \cdots, i_{j}, t=1,2, \cdots, k_{l}$, where $Q_{j m}^{(1)}$ operates on $x$ and $Q_{i l}^{(2)}$ operates on $y$. Then we define $D_{X}$ as the space function of ${ }_{2} D$ with the topology defined by the sets $N$. It is clear that the topology of $D_{X}$ is strictly weaker than the topology of ${ }_{2} D$.

THEOREM 2. Let $s: D_{X} \rightarrow V$ be the map which assigns to each $r \in D_{X}$ the linear map $U$ such that $r$ is the kernel representing $U$. Then $s$ is a topological isomorphism onto.

Proof. It is clear that $s$ is linear; we have already noted that $s$ is onto. Let $B$ be a bounded set in $D^{\prime}$ and $M_{1}$ a neighborhood of zero in $D$; we want to produce a neighborhood $N$ of zero in $D_{\times}$such that, for any $f \in N, T \in D^{\prime} s f(T) \in M_{1}$. Call $M_{2}$ the set of $g \in D$ such that $|S \cdot g| \leqq 1$ for all $S \in B$; since $D$ is reflexive, $M_{2}$ is a neighborhood of zero in D. Thus (see [2]) for each $j \geqq 0$ we can find two finite sequences of differential operators $Q_{j 1}^{(1)}, Q_{j 2}^{(1)}, \cdots, Q_{j i_{j}}^{(1)}$, and $Q_{j 1}^{(2)}, Q_{j 2}^{(2)}, \cdots, Q_{j k_{j}}^{(2)}$ so that, for $q=1,2, M_{q}$ contains the set of all $g \in D$ for which, for each $l$,

$$
\max _{x \geqq l}\left|\left(Q_{l m}^{(q)} g\right)(x)\right| \leqq 1
$$

for $m=1,2, \cdots, i_{j}$ if $q=1$, or for $s=1,2, \cdots, k_{j}$ if $q=2$.

Call $N$ the set of all $f \in D_{\times}$which satisfy (3). Then for any $f \in N$, $x \in R$, we have $f_{X_{1}=x} \in M_{2}$. Moreover, if $|x| \geqq j$, then we even have $Q_{j m}^{(1)} f_{X_{1}=x} \in M_{2}$ for $m=1,2, \cdots, i_{j}$. Thus, for any $S \in B$ and any $x$ with $|x| \geqq j$ we have, by the definition of $M_{2}$,

$$
\left|\left[Q_{j m}^{(1)} s f(S)\right](x)\right|=\left|S \cdot\left(Q_{j m}^{(1)} f\right)_{X_{1}=x}\right| \leqq 1
$$

for $m=1,2, \cdots, i_{j}$. This proves that $s f(S) \in M_{1}$ which shows that $s$ is continuous.

It remains to show that $s^{-1}$ is continuous; let $N$ be the neighborhood of zero in $D_{\times}$consisting of all $f \in D_{\times}$which satisfy (3). Call $M^{\prime}$ the set of $f \in D$ for which

$$
\max _{|x| \geqq j}\left|\left(Q_{j m}^{(1)} f\right)(x)\right| \leqq 1
$$

for all $j$ and for $m=1,2, \cdots, i_{j}$; set

$$
B^{\prime}=\left\{Q_{j t}^{(2)} \delta_{y}\right\}_{|y| \geqq j}
$$

for all $j$ and for $t=1,2, \cdots, k_{j}$. It is clear that $B^{\prime}$ is bounded in $D^{\prime}$ and that $M^{\prime}$ is a neighborhood of zero in $D$. Call $N^{\prime}$ the set of all 
$r \in D_{X}$ which represent elements $U \in V$ such that $U(T) \in M^{\prime}$ for all $T \in B^{\prime}$, so $N^{\prime}$ is the image in $D_{X}$ (under $s^{-1}$ ) of an open set in $V$. We want to show that $N^{\prime} \subset N$. Now, given any $r \in N^{\prime}$, if $r$ is the kernel representing $U \in V$, then for any $j, l$, and for $m=1,2, \cdots, i_{j}$, $t=1,2, \cdots, k_{l}$, if $|x| \geqq j,|y| \geqq l$, we have

$$
\left|\left(Q_{j m}^{(1)} Q_{l t}^{(2)} r\right)(x, y)\right|=\left|\left(Q_{j m}^{(1)} U\left(Q_{t t}^{(2)} \delta_{y}\right)\right)(x)\right| \leqq 1 .
$$

Thus, $N^{\prime} \subset N$ which concludes the proof of Theorem 2.

\section{BIBLIOGRAPHY}

1. L. Ehrenpreis, Some properties of distributions on Lie groups, to appear in Pacific Journal of Mathematics.

2. L. Schwartz, Theorie des distributions, vols. I and II, Paris, 1950-1951.

3. - Theorie des noyaux, Proceedings of the International Congress of Mathematicians, Cambridge, 1950, vol. I, pp. 220-230.

The Institute for Advanced Study 